\title{
“EL NIÑO OSCILACIÓN DEL SUR" Y ESTADO DE SALUD DE LAS IGUANAS MARINAS (Amblyrhynchus cristatus) EN GALÁPAGOS
}

\section{EL NIÑO - SOUTHERN OSCILLATION AND HEALTH STATUS OF GALAPAGOS MARINE IGUANA (Amblyrhynchus cristatus)}

\author{
Patricia Castillo-Briceño ${ }^{1}$, Cruz Márquez ${ }^{2}$, David Wiedenfeld ${ }^{3}$, Howard Snell $^{4}$ y Alberto Jaramillo ${ }^{5}$
}

\section{Resumen}

El Niño / Oscilación del Sur (ENOS) por interacción oceánico-atmosférica en el océano Pacífico tropical afecta al componente biológico. Estudios científicos sugieren que en iguanas marinas (Amblyrhynchus cristatus Bell, 1825) de Galápagos el impacto es negativo. La Fundación Charles Darwin para obtener información de largo plazo estableció un proyecto permanente de monitoreo en un sector de la isla Santa Cruz. El área de estudio abarca $6.1 \mathrm{~km}$ de costa desde Punta Núñez al sureste de la Isla hasta el muelle de la Estación Científica Charles Darwin.

Este trabajo analizó el estado de salud de la población de iguanas marinas en esta zona por niveles 0-5 (saludable a muerta), comparando entre los períodos ENOS (1997-98) y Post ENOS (2000-01). Se determinó la diferencia entre éstos, y la relación entre niveles de salud y condiciones ambientales (precipitación, temperatura superficial del mar y del aire). Los resultados mostraron una diferencia significativa $\left(\mathrm{z}_{0,05}=0.4477 ; \mathrm{P}<0.0001\right)$ en el nivel de salud de la población de iguanas marinas entre los dos períodos, pese a que los niveles de salud no presentaron relación directa $(\mathrm{r}<0.426)$ con las condiciones ambientales. Sin embargo, si las condiciones ambientales anómalas se mantienen la relación es directa $(\mathrm{r}=0.624)$, siendo notorio el deterioro del estado de salud después del sexto mes con temperatura superficial del mar $>23^{\circ} \mathrm{C}$ (promedio Post ENOS), que luego de un periodo igual en condiciones normales se recupera.

Palabras Clave: Amblyrhynchus cristatus, efecto de ENOS, estado de salud, temperatura superficial del mar, Galápagos.

\begin{abstract}
El Niño / Southern Oscillation (ENSO) affects biological components by its oceanicatmospheric interaction in the tropical Pacific ocean. For Galápagos marine iguanas (Amblyrhynchus cristatus Bell, 1825) scientific studies propose a negative impact. Charles Darwin Foundation, in order to acquire long term data, has established a permanent monitoring project in an area of Santa Cruz Island. This area has $6.1 \mathrm{~km}$ of coastal land (61 sampling stations), from Punta Núñez (SE of the island) to Charles Darwin Research Station dock.

This research shows the health state of the marine iguana population from this zone, considering six levels from 0 to 5 (healthy to dead), comparing ENSO (1997-98) and Post ENSO (2000-01) periods. Their differences and relation to health levels and environmental conditions (rain, superficial sea temperature and air temperature) were determined. Results showed significant differences $\left(\mathrm{z}_{0.05}=0.4477 ; \mathrm{P}<0.0001\right)$ in the health level of the marine iguana population between these periods, although health levels were not directly related $(\mathrm{r}<0.426)$ to environmental conditions. But if anomalous environmental conditions are maintained, a direct relationship $(r=0.624)$ is found. Health state diminishing was notorious after sixth months with superficial sea temperature higher than $23^{\circ} \mathrm{C}$ (Post ENSO average), and recovering was noticed after an equal period of normal conditions. Key words: Amblyrhynchus cristatus, ENSO effect, health state, sea superficial temperature, Galápagos.
\end{abstract}

\section{Introducción}

Las iguanas marinas (Amblyrhynchus cristatus Bell, 1825: Squamata, Iguanidae) endémicas de Galápagos donde están ampliamente distribuidas, con mayores concentraciones en las islas occidentales (Laurie, 1986), son los únicos saurios que dependen totalmente del mar para subsistir (Howick, 1977).

Están adaptadas morfológica, fisiológica y etológicamente a vivir en el borde costero. Ajustan su temperatura corporal con variaciones de postura
(Dowling, 1962), descansan y se reproducen en arrecifes de arena orgánica y lava (Carpenter, 1966). Ingieren agua de mar, eliminando la sal con saliva por las fosas nasales y se alimentan de algas, en especial durante bajamar, con técnicas de forrajeo submareal e intermareal (Wikelski et al., 1993). En febrero, época de baja producción algal y posterior a la temporada de apareamiento, los machos presentan desgaste físico; hacia junio se recuperan, pues dedican más tiempo al 
forrajeo y los nutrientes del mar favorecen la producción algal (Drent et al., 1999).

ENOS es un fenómeno natural de interacción oceánico-atmosférica relacionado con la intensidad y dirección de los vientos alisios, temperatura superficial del mar (TSM), presión atmosférica superficial, temperatura del aire (TA), pluviosidad y nivel del mar. A lo largo del Pacífico tropical se manifiesta con la fluctuación de las fases cálida ("El Niño") y fría ("La Niña" o "El Viejo") de la TSM, con repercusiones sobre el componente biológico. En Galápagos implica mayor alimento disponible para la fauna terrestre, pero una disminución drástica para la marina (Laurie, 1984). Esta disminución puede causar mortalidad de iguanas marinas por inanición como la registrada durante ENOS 1982-83 (Cooper \& Laurie, 1987), debido a que las algas verdes y rojas que son su alimento habitual desaparecen y son reemplazadas por algas pardas de difícil digestión, afectando a más del 90\% de la población (Laurie, 1990).

Por esta razón, en 1997, la Fundación Charles Darwin (FCD) inició un programa de monitoreo permanente para determinar la interacción iguanas marinas-ambiente en función del evento ENOS y su influencia en la permanencia de esta población.

\section{Materiales y métodos}

El área de estudio está ubicada al sur de la isla Santa Cruz en Galápagos y abarca $6.1 \mathrm{~km}(3.5 \%$ del perímetro total de la Isla). Consta de 61 estaciones de 100 m cada una, desde la entrada de Punta Núñez (sureste de la isla) con la estación $\mathrm{N}^{\circ} 100\left(0^{\circ} 44^{\prime} 45^{\prime} \mathrm{S}\right.$, $\left.90^{\circ} 15^{\prime} 19^{\prime \prime} \mathrm{O}\right)$ hasta el muelle de la Estación Científica Charles Darwin (ECCD) con la estación $\mathrm{N}^{\circ} 6100$ (044’36’'S, 90¹8'17’O) (Figura 1).

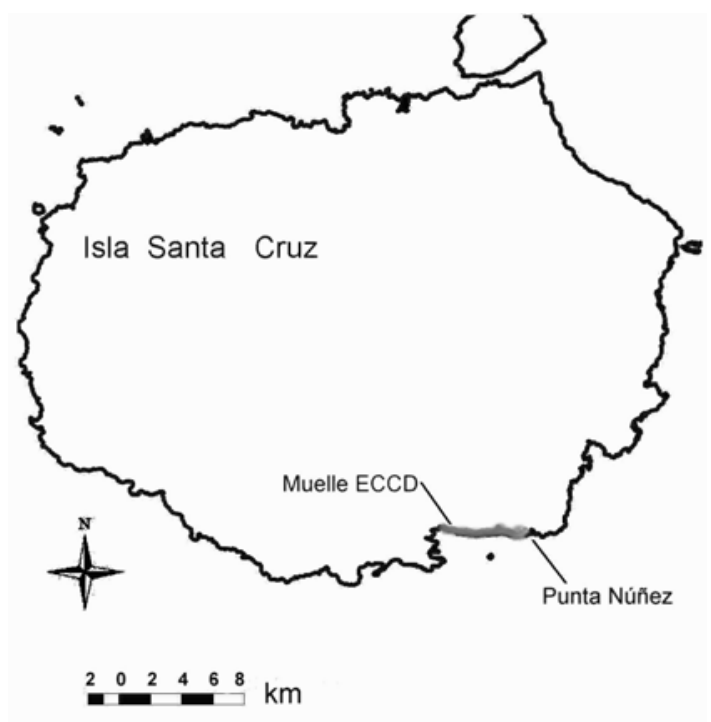

Figura 1. Mapa del área de estudio. La zona de muestreo (acantilado) está resaltada en gris.
El clima local es biestacional: caliente con lluvia representativa (Enero-Mayo) y frío con llovizna ligera $\mathrm{y}$ persistente, frecuentemente nublado (JunioDiciembre). En la costa el suelo está constituido principalmente de conchilla y roca volcánica, con presencia de manglar y plantas de ribera como Sesuvium, y macroalgas en la zona intermareal.

Los monitoreos son mensuales, las iguanas son capturadas aleatoriamente para medir: longitud corporal (de boca a cloaca), ancho de cabeza (en el borde anterior de los orificios auditivos) y peso; información ingresada a la base de datos. El nivel de salud se determinó por el aspecto externo del animal (Tabla 1).

Tabla 1. Descripción de los niveles de salud en iguanas marinas.

\begin{tabular}{|c|c|}
\hline $\begin{array}{c}\text { Nivel } \\
\text { de } \\
\text { salud }\end{array}$ & Características \\
\hline $\mathbf{0}$ & Saludable y robusta \\
\hline 1 & $\begin{array}{l}\text { Base de la cola delgada, huesos de cadera } \\
\text { ligeramente prominentes }\end{array}$ \\
\hline 2 & $\begin{array}{l}\text { Huesos de cadera y rodillas perfilados, costillas } \\
\text { poco prominentes }\end{array}$ \\
\hline 3 & $\begin{array}{l}\text { Condición semiesquelética con capacidad de } \\
\text { desplazamiento }\end{array}$ \\
\hline 4 & $\begin{array}{l}\text { Condición semiesquelética sin capacidad de } \\
\text { desplazamiento }\end{array}$ \\
\hline 5 & $\begin{array}{l}\text { Muerte por deterioro de salud, especialmente } \\
\text { inanición }\end{array}$ \\
\hline
\end{tabular}

Para establecer los efectos del evento ENOS sobre el estado de salud de la población de iguanas marinas, se observaron 1648 animales entre los periodos ENOS 1997-98 (condiciones anómalas) y Post ENOS 200001 (condiciones normales), de Noviembre del primer año a Diciembre del segundo en cada período. Con la prueba de U (Mann-Whitney) se comparó las series del nivel de salud para los dos periodos infiriendo al estadístico $\mathrm{z}_{\alpha=0.05}$. El nivel de salud y su relación con las condiciones ambientales: TSM, TA, precipitación y frecuencia acumulada de $\mathrm{TSM}>23^{\circ} \mathrm{C}$ (promedio durante el periodo Post ENOS) se analizó por regresión lineal y su coeficiente de correlación crítico $\left(\mathrm{r}_{\mathrm{t}}=0.426\right)$.

\section{Resultados}

El nivel de salud de las iguanas marinas durante ENOS 1997-98 fue 2.07 mientras en el período Post ENOS 2000-01 fue 0.24. El pico máximo en ambos casos se presentó en Julio con 3.17 y 0.46 respectivamente, mientras los mínimos ocurrieron en Diciembre 1998 (0.20) y Noviembre 2000 (0.04). El estado de salud significativamente diferente $\left(\mathrm{z}_{0.05}=0.4477 ; \mathrm{P}<0.0001\right)$ entre estos periodos, fue mejor en la época normal (Figura 2; Tabla 2). 


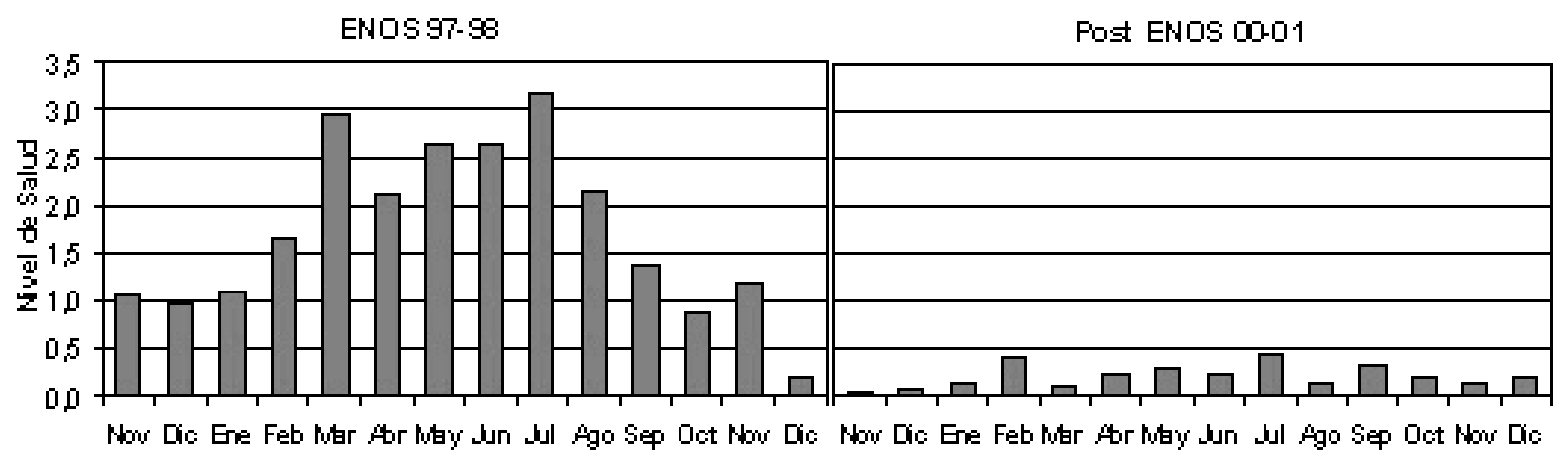

Figura 2. Sucesión mensual del nivel de salud para los periodos ENOS 1997-98 y Post ENOS 2000-01.

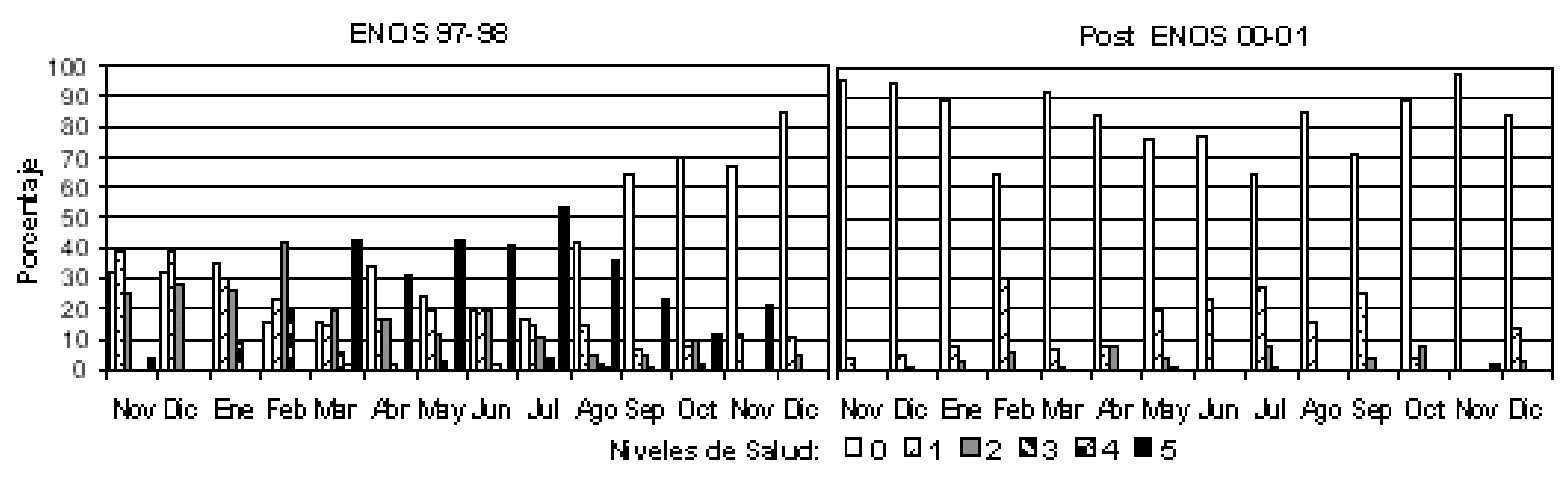

Figura 3. Distribución porcentual de los niveles de salud para los periodos ENOS 1997-98 y Post ENOS 2000-01

Tabla 2. Niveles de salud promedio de ENOS 1997-98 y Post ENOS 2000-01.

\begin{tabular}{lcc}
\hline & \multicolumn{2}{c}{ Nivel de Salud } \\
\cline { 2 - 3 } Mes & $\begin{array}{c}\text { ENOS } \\
\mathbf{( 1 9 9 7 - 9 8 )}\end{array}$ & $\begin{array}{c}\text { Post ENOS } \\
\mathbf{( 2 0 0 0 - 0 1 )}\end{array}$ \\
\hline Noviembre & 1.07 & 0.04 \\
\hline Diciembre & 0.96 & 0.07 \\
\hline Enero & 1.09 & 0.13 \\
\hline Febrero & 1.67 & 0.41 \\
\hline Marzo & 2.94 & 0.09 \\
\hline Abril & 2.11 & 0.24 \\
\hline Mayo & 2.63 & 0.29 \\
\hline Junio & 2.65 & 0.23 \\
\hline Julio & 3.17 & 0.46 \\
\hline Agosto & 2.14 & 0.15 \\
\hline Septiembre & 1.38 & 0.32 \\
\hline Octubre & 0.87 & 0.19 \\
\hline Noviembre & 1.17 & 0.12 \\
\hline Diciembre & 0.20 & 0.19 \\
\hline Promedio & $\mathbf{2 . 0 7}$ & $\mathbf{0 . 2 4}$
\end{tabular}

La mayor parte de la población durante el ENOS 1997-98 estuvo en los niveles 0 (35\%) y 5 (30\%), mientras en el lapso Post ENOS 2000-01 el $81 \%$ correspondió al nivel $\mathbf{0}$ (Figura 3; Tabla 3). El histórico de los niveles de salud desde 1997 hasta 2003 mostró que entre 1997 y 1998 hubo el mayor deterioro, mientras que el mejor estado de salud ocurrió entre el 2000 y 2001 (Figuras 4 y 5).

Respecto al nivel de salud de acuerdo con la estructura poblacional, durante ENOS 1997-98 los machos adultos fueron más afectados, con mayor mortalidad; situación opuesta a la del periodo Post ENOS 2000-01 en el que fueron los juveniles (Figura 6).

Entre el estado de salud y la frecuencia acumulada de TSM $>23^{\circ} \mathrm{C}$ se presentó una relación directa $(\mathrm{r}=0.624)$ con tendencia ascendente. Pero con las condiciones climáticas en general: TSM (Figura 7), TA (Figura 8) y precipitación (Figura 9), no hubo correlación $(\mathrm{r}<0.426)$. El deterioro del estado de salud de las iguanas marinas fue evidente a partir del sexto mes con TSM $>23^{\circ} \mathrm{C}$, mientras en la etapa Post ENOS 2000-01 no se identificó este patrón, pero se observó que a partir del sexto mes consecutivo en condiciones normales la recuperación es patente (Figura 10).

\section{Discusión}

La elevada mortalidad de iguanas marinas registrada en Punta Núñez durante el evento ENOS 1997-98 con respecto al periodo Post ENOS 2000-01 
en que fue muy baja; es comparable con la mortalidad durante ENOS 1982-84 registrada en la mayor parte de las islas Galápagos con respecto a los períodos previo 1981-82 (Laurie, 1984; Cooper \& Laurie, 1987) y posterior (Buttemer \& Dawson, 1993) cuyas condiciones ambientales fueron normales. Lo que supone una relación entre el deterioro del estado de salud de esta población y las condiciones ambientales anómalas características de ENOS, especialmente en la parte marina.

Tabla 3. Distribución porcentual de los niveles de salud en los periodos ENOS 1997-98 y Post ENOS 2000-01.

\begin{tabular}{ccccc}
\hline \multirow{2}{*}{$\begin{array}{c}\text { Nivel de } \\
\text { Salud }\end{array}$} & \multicolumn{2}{c}{ ENOS 1997-98 } & \multicolumn{2}{c}{ ENOS 2000-01 } \\
\cline { 2 - 5 } & Individuos & $\mathbf{\%}$ & Individuos & $\mathbf{\%}$ \\
\hline $\mathbf{0}$ & 412 & 35.1 & 734 & 80.7 \\
\hline $\mathbf{1}$ & 200 & 17.0 & 137 & 15.1 \\
\hline $\mathbf{2}$ & 165 & 14.0 & 39 & 4.3 \\
\hline $\mathbf{3}$ & 40 & 3.4 & 0 & 0.0 \\
\hline $\mathbf{4}$ & 3 & 0.3 & 0 & 0.0 \\
\hline $\mathbf{5}$ & 355 & 30.2 & 0 & 0.0 \\
\hline Totales & $\mathbf{1 1 7 5}$ & $\mathbf{1 0 0}$ & $\mathbf{9 1 0}$ & $\mathbf{1 0 0}$ \\
\hline
\end{tabular}

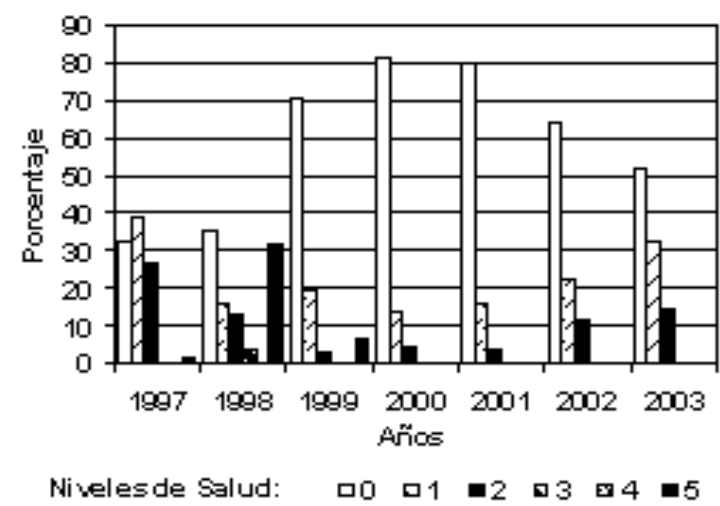

Figura 4. Distribución anual de los niveles de salud desde 1997 hasta el 2003.

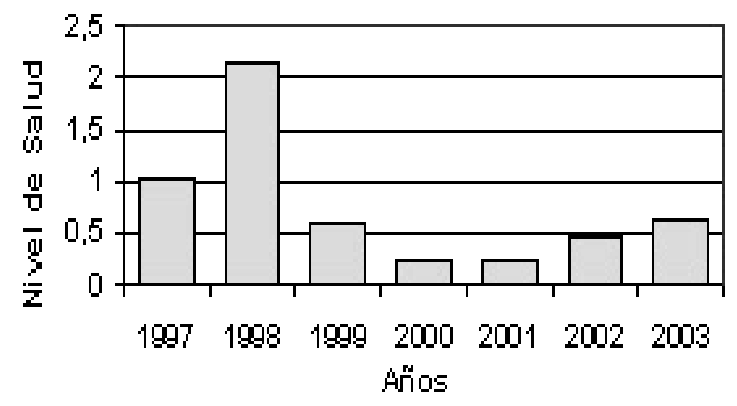

Figura 5. Sucesión anual del nivel de salud desde 1997 hasta el 2003.

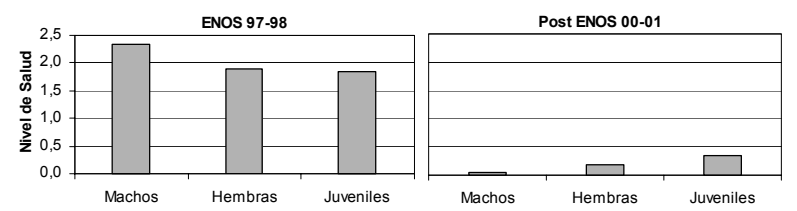

Figura 6. Niveles de salud vs. estructura poblacional para los periodos ENOS 1997-98 y Post ENOS 200001 .

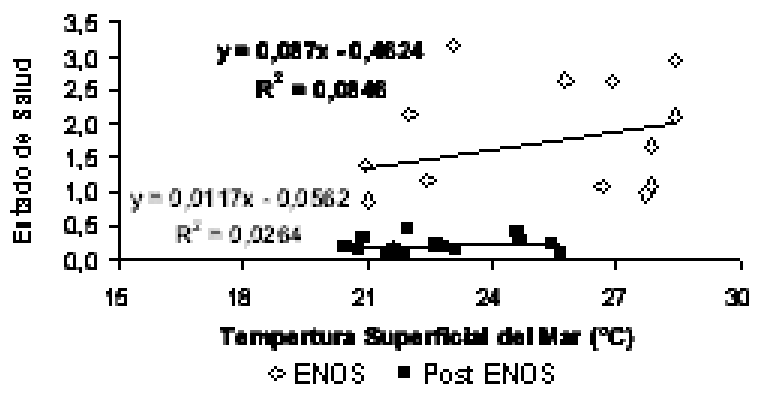

Figura 7. Niveles de salud vs. temperatura superficial del mar en los periodos ENOS 1997-98 y Post ENOS 2000-01.

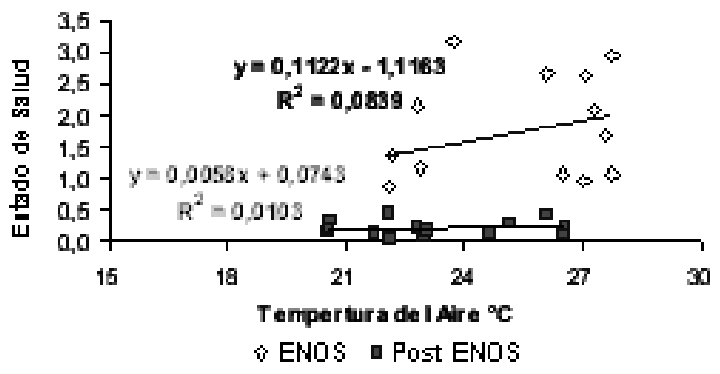

Figura 8. Niveles de salud vs. temperatura del aire en los periodos ENOS 1997-98 y Post ENOS 2000-01.

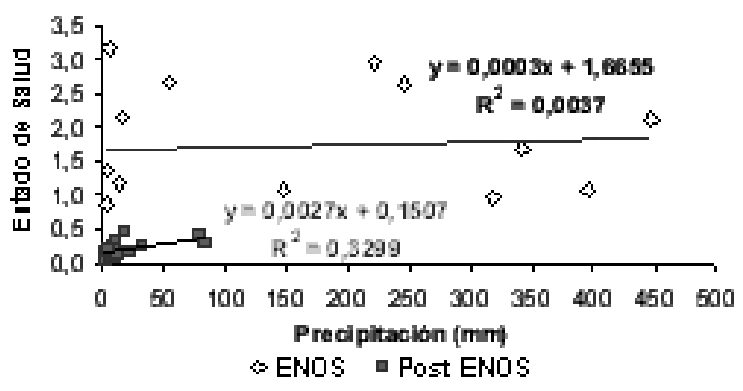

Figura 9. Niveles de salud vs. precipitación en los periodos ENOS 1997-98 y Post ENOS 2000-01. 


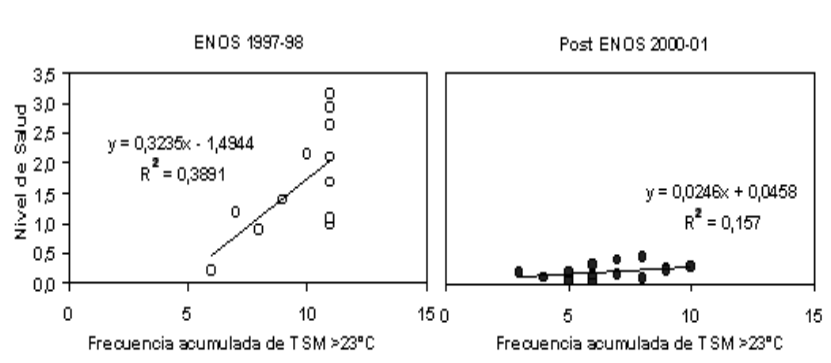

Figura 10. Niveles de salud vs. frecuencia acumulada de la temperatura superficial del mar $(\mathrm{TSM})>23^{\circ} \mathrm{C}$ en los periodos ENOS 1997-98 y Post ENOS 2000-01.

Durante El Niño en Galápagos la TSM (promedio normal $18^{\circ} \mathrm{C}$ ) alcanza los $32^{\circ} \mathrm{C}$, las corrientes frías, salinidad y afloramientos de nutrientes decrecen (Wikelski et al., 1993), con la subsiguiente alteración ecológica. Desaparecen la mayoría de algas de aguas frías comunes durante los períodos normales (Cooper \& Laurie, 1987), alimento habitual de iguanas marinas, quienes presentarán peso bajo y alta mortalidad por inanición (Laurie, 1984). Cuando las condiciones ambientales se normalizan inicia un proceso de restauración de la flora algal en tres fases: disminución de biomasa algal con mortalidad masiva de herbívoros por falta de alimento, menor presión de forrajeo con recuperación de algunas algas e incremento de biomasa algal que estimula la repoblación de herbívoros (Garske, 2002). Proceso que coincide con los resultados del presente estudio, donde el estado de salud deteriorado de las iguanas marinas durante las condiciones anómalas del evento ENOS 1997-98, mejora progresivamente en el lapso de tres años de condiciones ambientales normales hasta el periodo Post ENOS 2000-01 en que alcanza su mejor nivel (Figuras 4 y 5).

El mayor deterioro del estado de salud en machos adultos, con respecto a hembras adultas y juveniles durante ENOS 1997-98, coincide con la alta mortalidad de iguanas de mayor tamaño en ENOS 1982-83. Generalmente en organismos forrajeros el tamaño es inversamente proporcional a la eficiencia alimentaria (Wikelski et al., 1993), aunque también influyen la disponibilidad de alimento y estrategia de forrajeo (Laurie, 1984). Durante ENOS el mayor daño ocurre en las zonas intermareal y submareal someras, donde la mitigación de anomalías en la TSM es menor (Garske, 2002), lo cual afecta más a los machos adultos que se alimentan en ambas zonas (especialmente submareal), mientras hembras y juveniles lo hacen en la zona intermareal. También el metabolismo influye, cuando la temperatura del medio es de $25^{\circ} \mathrm{C}$ el consumo de oxígeno es bajo lo cual aprovechan para alimentarse y optimizar energía, pero al aumentar la temperatura durante El Niño el consumo se incrementa (mayor consumo de oxígeno de $30^{\circ} \mathrm{C}$ a $40^{\circ} \mathrm{C}$ ) afectando la alimentación bajo el agua (Bennet et al., 1975).

La ausencia de relación significativa entre las condiciones ambientales típicas de un fenómeno ENOS y los niveles de salud se deberían a que el efecto es indirecto. La accesibilidad al alimento sería un factor determinante en el estado de salud de la población de iguanas marinas, en función del impacto de estas condiciones mantenidas en el tiempo sobre la producción algal.

La correlación entre niveles de salud y frecuencia acumulada de la $\mathrm{TSM}>23^{\circ} \mathrm{C}$ significaría que la verdadera causa que afecta a la salud de la población es la extensión temporal de temperaturas elevadas (Figura 10). Estas condiciones sostenidas al afectar la disponibilidad algal se verían reflejadas en el progresivo deterioro del estado de salud de las iguanas marinas. De esta forma el nivel de salud de las iguanas actuaría como indicador de las condiciones ambientales anómalas preexistentes, pero no de las presentes. A la vez quedaría expuesta la posibilidad de que para el estado de salud, la duración de las condiciones atípicas características de fenómenos naturales como el evento ENOS tendría un mayor impacto que la intensidad en sí de los mismos.

\section{Conclusiones}

El impacto negativo sobre la población de iguanas marinas del fenómeno natural ENOS puede ser exitosamente compensado en un tiempo relativamente corto, si las condiciones del entorno se normalizan y no existe interferencia de otros factores, situación observada durante ENOS 1982-83 y se corrobora en el presente estudio, donde el historial del estado de salud muestra una recuperación total en el periodo Post ENOS 2000-01 con respecto a ENOS 1997-98.

El notorio impacto de las condiciones ambientales de este evento sobre el estado de salud de las iguanas, resalta su alta capacidad de repoblación y recuperación, alcanzando un nuevo estado estable de salud en condiciones normales. Esta interacción como medio de selección natural garantiza la permanencia temporal de la especie, pues renueva periódicamente la población y la restaura con los individuos mejor adaptados, en función de su capacidad de supervivencia.

Las condiciones ambientales anormales mantenidas causan el progresivo deterioro del estado de salud de las iguanas marinas, en consecuencia para esta población la duración de las condiciones atípicas es un factor crítico tanto o más importante que las anomalías en sí, pues recién a partir de seis meses las repercusiones del evento son significativas. Es así que la extensión temporal de las anomalías es directamente proporcional al deterioro de la salud de estos organismos y afecta en mayor grado que la intensidad con que se presentan. De esta forma el nivel de salud de las iguanas marinas no representa las condiciones 
ambientales anómalas o normales en curso, sino que estará siempre referido a las condiciones previas sostenidas durante un lapso mínimo de seis meses.

\section{Agradecimiento}

Nuestra sincera gratitud a las voluntarias y voluntarios de colegios, universidades y profesionales que nos apoyaron en la toma de datos durante los muestreos. A las organizaciones internacionales que canalizaron sus fondos a través de la FCD y a la misma por el apoyo logístico a través del Área de Investigación y Conservación Marina. Finalmente, a todas y cada una de las personas que han participado en los monitoreos y nos ayudaron durante la realización del presente estudio.

\section{Literatura citada}

Bennet A.F., Dawson W.R. \& Bartholomew G.A. 1975. Effects of Activity and Temperature on Aerobic and Anaerobic Metabolism in the Galapagos Marine Iguana. En: J. comp. Physiol. 100: 317-329. Springer-Verlag.

Buttemer W.A. \& Dawson W.R. 1993. Temporal pattern of foraging and microhabitat use by Galápagos marine iguanas, Amblyrhynchus cristatus. Oecology. 96: 56-64. Springer-Verlag.

Carpenter C.C. 1966. The Marine Iguana of the Galápagos Islands, Its behavior and Ecology. Proc. Calif. Acad. Sci. 34 (6): 329-376.

Cooper J.E. \& Laurie W.A. 1987. Investigation of Deaths in Marine Iguanas (Amblyrhynchus cristatus) on Galapagos. J. Comp. Path. 97 (2): 129-136.

\footnotetext{
${ }^{1}$ Fundación Charles Darwin

ECCD, Puerto Ayora, Galápagos-Ecuador.

Pontificia Universidad Católica del Ecuador, Sede Manabí km 8 1/2 vía Bahía de Caráquez-Chone Villas del IESS Mz D-33, Manta-Ecuador. Correo electrónico: patcb@latinmail.com; pacabri@yahoo.com

2 (Fundación Charles Darwin)

ECCD, Puerto Ayora, Galápagos-Ecuador. Casilla Postal: 17-01-3891. Correo electrónico: marquez@fcdarwin.org.ec

3 (Fundación Charles Darwin). Correo electrónico: dwiedenfeld@fcdarwin.org.ec

4 (Fundación Charles Darwin). Correo electrónico: howard@fcdarwin.org.ec

5 (Fundación Charles Darwin). Correo electrónico: albertoj@fcdarwin.org.ec
}

Dowling R.G. 1962. Sea Dragons of the Galapagos: The Marine Iguanas. En: Animal Kingdom. 65: 169-174.

Drent J., Van Marken Lichtenbelt D. \& Wikelski M. 1999. Effects of foraging mode and season on the energetics of the Marine iguana, Amblyrhynchus cristatus. En: Funstional Ecology. British Ecological Society. 13: 493-499.

Garske L.E. 2002. Macroalgas Marinas. En: Reserva Marina de Galápagos. Línea Base de la Biodiversidad. Eva Danulat y Grahm J. Edgar (editores).: 419-431. Estación Científica Charles Darwin / Servicio del Parque Nacional. Santa Cruz, Galápagos, Ecuador.

Howick G. 1977. The Uniqueness of the Marine Iguana. Student Report for Colorado State Univ., CDRS Library. : 1-7.

Laurie W.A. 1984. El Niño Causa Estragos Nunca Vistos en la Población de Iguanas Marinas. La Sirena. 25: 11-14.

Laurie W.A. 1986. Effects of the 1982-83 El Niño Sea Warming on Marine Iguana (Amblyrhynchus cristatus Bell, 1825) Populations on Galápagos. Report, CDRS Library. : 1-18.

Wikelski M., Gall B. \& Trillmich F. 1993. Ontogenetic Chances in Food Intake and Digestion Rate of the Herbivorous Marine Iguana (Amblyrhynchus cristatus Bell). Oecología. 94: 373-379. 